\title{
Overcoming immunotherapy resistance in non-small cell lung cancer (NSCLC) - novel approaches and future outlook
}

\author{
Lena Horvath', Bernard Thienpont ${ }^{2}$, Liyun Zhao ${ }^{2}$, Dominik Wolf ${ }^{1,3}$ and Andreas Pircher ${ }^{1 *}$ (D)
}

\begin{abstract}
Immunotherapy $(I O)$ has revolutionized the therapy landscape of non-small cell lung cancer (NSCLC), significantly prolonging the overall survival (OS) of advanced stage patients. Over the recent years $1 \mathrm{O}$ therapy has been broadly integrated into the first-line setting of non-oncogene driven NSCLC, either in combination with chemotherapy, or in selected patients with PD-L1 ${ }^{\text {high }}$ expression as monotherapy. Still, a significant proportion of patients suffer from disease progression. A better understanding of resistance mechanisms depicts a central goal to avoid or overcome IO resistance and to improve patient outcome.

We here review major cellular and molecular pathways within the tumor microenvironment (TME) that may impact the evolution of $\mathrm{IO}$ resistance. We summarize upcoming treatment options after $\mathrm{IO}$ resistance including novel $1 \mathrm{O}$ targets (e.g. RIG-I, STING) as well as interesting combinational approaches such as 10 combined with anti-

angiogenic agents or metabolic targets (e.g. IDO-1, adenosine signaling, arginase). By discussing the fundamental mode of action of $I O$ within the TME, we aim to understand and manage $I O$ resistance and to seed new ideas for effective therapeutic 10 concepts.
\end{abstract}

Keywords: NSCLC, Immunotherapy resistance, Tumor microenvironment heterogeneity, Targeted therapy

\section{Background}

Immunotherapy (IO) and particularly immune checkpoint inhibitors (ICI), including programmed death receptor 1 (PD-1) and PD-ligand 1 (PD-L1) inhibitors have revolutionized the treatment landscape of nonsmall cell lung cancer (NSCLC). Previously unanticipated long-term responses in advanced stage disease have been accomplished, with a 5 year overall survival (OS) of $20 \%$ in unselected and up to $40 \%$ in PD-L1 ${ }^{\text {high }}$ expressing patients [1].

Despite the striking clinical improvements, the majority of patients eventually fails to respond to ICI therapy due to the evolution of primary or secondary resistance.

\footnotetext{
* Correspondence: andreas.pircher@i-med.ac.at

'Internal Medicine V, Department of Hematology and Oncology, Medical University Innsbruck, Anichstraße 35, 6020 Innsbruck, Austria

Full list of author information is available at the end of the article
}

Prospective clinical studies to demonstrate treatment strategies following progression on IO therapy are still lacking.

Various IO resistance mechanisms have been characterized, involving tumor cell intrinsic as well as environmental resistance patterns. The tumor microenvironment (TME) plays a critical role by influencing both extrinsic and intrinsic resistance pathways. A better understanding of the heterogenous TME will set stage for further optimizing strategies and guide new avenues in future IO treatment stratification.

This review discusses the multitude of novel preclinical and clinical treatment approaches that aim to overcome IO resistance in NSCLC. The complexity of cellular and molecular alterations within the immunosuppressive TME build the fundament for designing rational and synergistic combination therapies that lower the risk of resistance and prolong benefit from IO therapy. 


\section{Immunopathology of NSCLC and evolution of IO resistance}

IO resistance mechanisms result from the constantly evolving interactions between cancer cells and the surrounding cell populations within the TME, including immune cells, cancer-associated fibroblasts (CAF) and tumor endothelial cells (TEC) (Fig. 1). The following section recapitulates the basic characteristics of the immunogenic TME, particularly focusing on IO responseor resistance-mediating mechanisms and biomarkers.

\section{Immune checkpoints}

Immune checkpoints (IC) play a central role in negative regulation of $\mathrm{T}$ cell reactivity and their inhibition via monoclonal antibodies can unleash $\mathrm{T}$ cell-triggered antitumor immune responses. The best studied IC are PD-1 and cytotoxic $\mathrm{T}$ lymphocyte antigen 4 (CTLA-4). PD-1 is broadly expressed on $\mathrm{CD}^{+} \mathrm{T}$ lymphocytes, regulatory $\mathrm{T}$ cells (Treg) and natural killer (NK) cells and modulates $\mathrm{T}$ cell activity via interaction with its ligand (PD-L1) in the TME (Fig. 2). CTLA-4 is expressed on $\mathrm{CD}^{+}$and $\mathrm{CD}^{+}{ }^{+} \mathrm{T}$ lymphocytes and Treg and regulates early naïve $\mathrm{T}$ cell activation in secondary lymphoid organs $[3,4]$. Other IC are constantly being discovered and under investigation for their clinical utility as druggable IC (e.g. TIM-3, LAG-3 or TIGIT).

\section{Lymphocytes of the tumor microenvironment $T$ lymphocytes}

Tumor infiltrating $\mathrm{T}$ lymphocytes (TIL) play a major role in antitumor immune responses within the TME

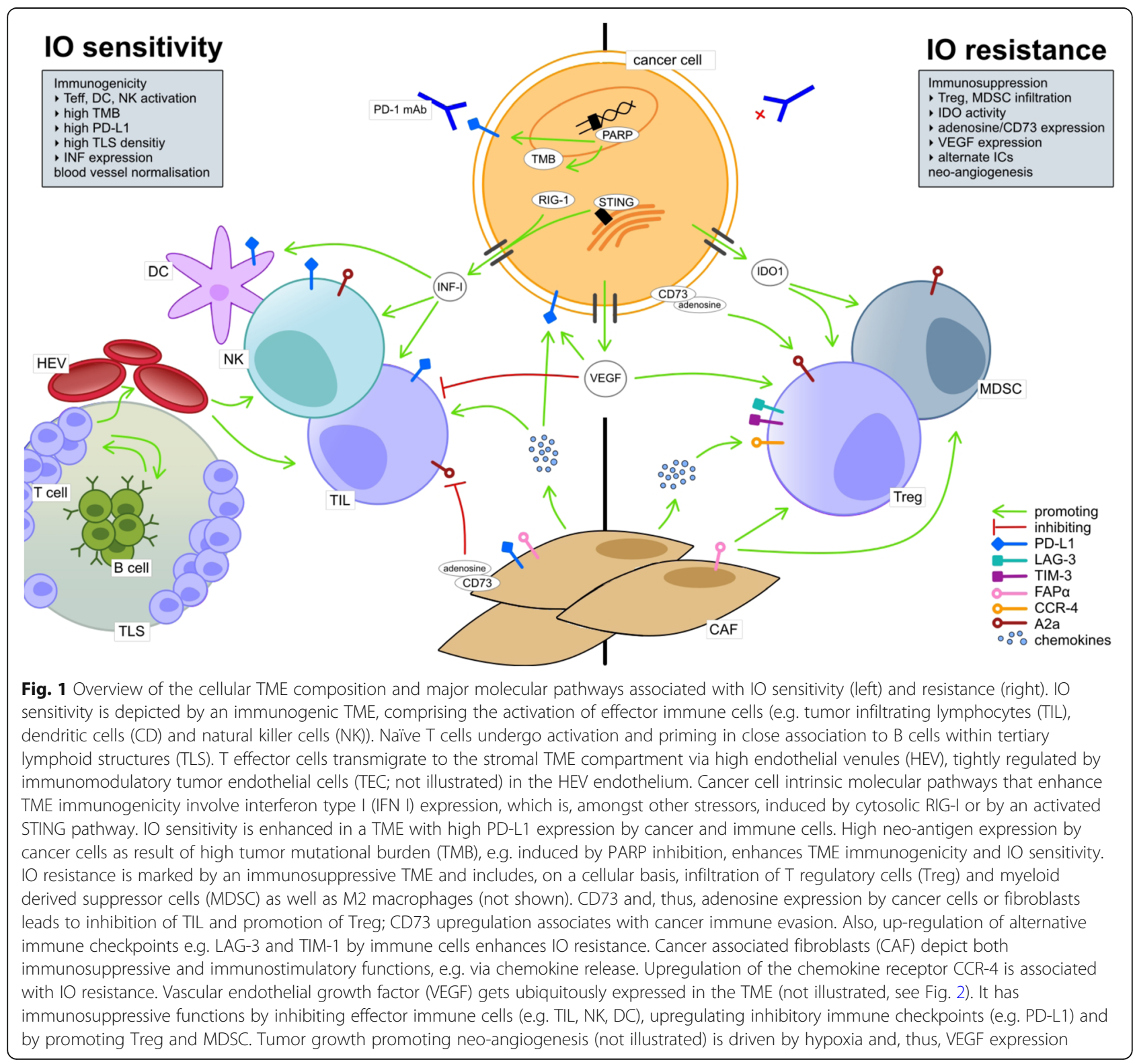




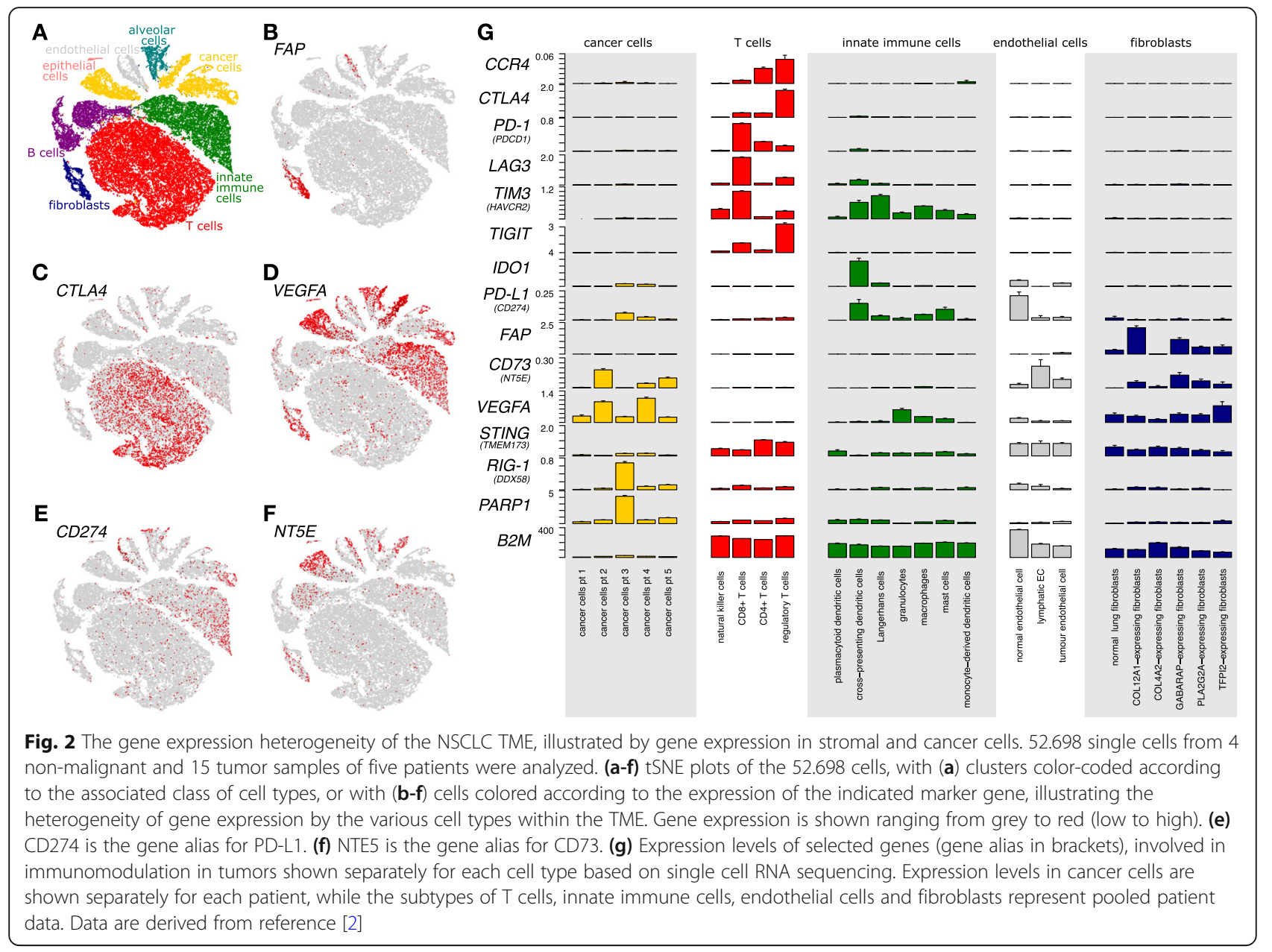

[5]. The phenotype of $\mathrm{T}$ cell infiltration varies strongly between different tumors in terms of quantity and distribution and is associated with IO efficacy. Classifying tumors based on the cytotoxic $\mathrm{T}$ cell infiltration phenotype might help to rationally guide treatment stratification [5].

\section{Tertiary lymphoid structures}

In chronically inflamed areas such as tumors, B and $\mathrm{T}$ lymphocytes are frequently organized in ectopic lymphoid aggregates, so-called tertiary lymphoid structures (TLS), where they convert to effector cells upon antigen presentation. The cellular organization ranges from simple lymphocyte clusters (immature TLS) to highly sophisticated structures (mature TLS) [6, 7]. High endothelial venules (HEVs) are found nearby and promote lymphocyte extravasation [8]. TLS display a surrogate marker of prompt immune responses that actively modulate anticancer immunity [7]. High TLS density associates with a favorable prognosis in many cancer types, including NSCLC [9] and TLS may also enhance IO efficacy [6]. Preclinical studies demonstrated beneficial effects of therapeutic TLS neogenesis on anti-cancer immune responses [10-12].

\section{B lymphocytes}

Tumor infiltrating B cells harbor both immunostimulatory [13] and immunosuppressive [14] functions and their effect on IO efficacy is increasingly appreciated. Especially those B cells located in mature TLS may exhibit immunostimulatory functions by closely interacting with local $\mathrm{T}$ cells, thereby enhancing anti-cancer immunity. This hypothesis is indirectly supported by the observation that intra-tumoral B cells are linked to a favorable IO response $[7,15,16]$.

\section{Tumor Mutational Burden (TMB)}

Somatic mutations in the cancer genome, such as in DNA repair genes including mismatch repair (MMR), homologous recombination (HR) or polymerase epsilon (POLE) increase tumor mutational and neoantigen burden, which has been linked to greater TIL density and enhanced ICI efficacy [17-19]. This observation is clinically underscored as mutagen-driven cancer types (e.g. 
melanoma, NSCLC) typically show high initial ICI responses [17]. Moreover, components of the major histocompatibility complex I (MHC I) such as B2M are often downregulated (Fig. 2), hence curbing neo-epitope presentation to T cells [20]. Antigen presentation pathways can also be inactivated through mutations (e.g. B2M is mutated or deleted in about 5\% of lung cancers) [21] and also other pathway members are inactivated [22]. Importantly, IO may increase the frequency of such mutations $[19,23,24]$ suggesting an active immune-editing of cells failing to present neo-epitopes.

Concerning TMB as predictive biomarker of ICI response, clinical trials report divergent results, possibly due to technical issues with TMB assessment (e.g. use of inhomogenous cut-off values) [25]. On the one hand, high TMB was the strongest variable linked to benefit of combined PD-1 plus CTLA-4 blockade in NSCLC and TMB was independent of PD-L1 expression [26]. Accordingly, pembrolizumab was recently FDA-approved in $\mathrm{TMB}^{\text {high }}$ advanced solid cancers $(\geq 10$ mutations/ megabase) in response to results from KEYNOTE-158. In contrast, in the complex multi-arm CheckMate227 trial testing ipilimumab plus nivolumab versus chemotherapy or nivolumab plus chemotherapy in NSCLC, neither TMB nor PD-L1 expression could segregate therapy responsiveness [27]. Concerning CTLA4-specific biomarkers, different genomic signatures were correlated with enhanced clinical outcome $[28,29]$, however none have been translated into clinical practice yet.

\section{PD-L1 expression in the TME}

Cancer cells can overexpress PD-L1 upon type I interferon (IFN I) stimulation [30] to evade cytotoxic immune responses. Immune cells, including Treg, myeloidderived suppressor cells (MDSC), dendritic cells (DC) and TEC can similarly upregulate PD-L1 upon inflammatory signals (especially by IFNs) fostering an immunosuppressive TME [31]. Interestingly, myeloid cells show markedly higher PD-L1 expression than cancer cells or lymphocytes (Fig. 2) and especially extratumoral PD-L1 expressing myeloid cells, e.g. in tumor draining lymph nodes, might be essential for ICI response [31]. A preclinical study demonstrated that myeloid progenitors that accumulate during cancer-driven emergency myelopoiesis (in bone marrow, spleen and tumor site) show both PD-L1 and particularly prominent PD-1 expression. Selective deletion of myeloid-specific PD-1 by targeting the $P d c d 1$ gene effectively suppressed tumor growth in several tumor models by mediating antitumor immunity (enhanced $\mathrm{T}$ effector memory cells) despite preserved T cell-specific PD-1 expression. These data underline the important role of myeloid-intrinsic effects in regulating anti-tumor immunity [32].
Clearly, PD-L1 expression is necessary to achieve adequate responses to PD-1/PD-L1 blockade and numerous studies associated high tumor cell PD-L1 expression with better outcomes to anti-PD-1/PD-L1 monotherapy in NSCLC. Controversially, some patients with very low or even absent PD-L1 expression show durable responses [33], an observation currently lacking a mechanistic explanation see 2.4.1. Besides cancer cells, also PD-L1 positive immune cells may exert a predictive value. In the Impower110 trial, presence of PD-L1 positive TIL significantly associated with enhanced OS in patients treated with atezolizumab [34]. These results are in line with other tumor entities (e.g. bladder and breast cancer).

\section{$P D-L 1$ is not yet a robust biomarker}

So far, clinical trials considered tumor PD-L1 expression as the most robust and reproducible biomarker, and clinical NSCLC guidelines are based on this. However, PD-L1 immunohistochemistry (IHC) has several limitations (e.g. biopsies from primary versus metastatic lesions, different detection antibodies and cut-offs, staining procedures) and this may contribute to the above-mentioned controversial observations. Moreover, the TME is highly heterogenous and a single core biopsy only depicts one spatial tumor component, hence some patients may be PD-L1 negative in one biopsy and PDL1 positive in other tumor areas. This also explains quantification errors in tissue-based biomarkers. One approach to resolve the limitation of spatial resolution involves PET-based PD-L1 imaging with zirconium-89labeled atezolizumab. Interestingly, pre-treatment tumor PET signal was shown to better correlate with clinical treatment responses than IHC or RNA-sequencing based predictive biomarker-detection [35].

\section{Tumor-associated macrophages}

Tumor-associated macrophages (TAM) are an abundant cell type within the TME and despite growing research, their role in cancer progression remains ambiguous. Along a functional scale, TAM polarize to either M1 or M2 phenotypes in response to environmental cues, including metabolic changes (e.g. cyclic hypoxia, nitric oxide) [36, 37]. The classically activated M1 phenotype is stimulated upon type $1 \mathrm{~T}$ helper cell (Th1)-produced IFN$\gamma$ or Toll-like receptor (TLR) ligands such as microbiotaderived lipopolysaccharide (LPS) and is characterized by phagocytic, cytotoxic and antigen-presenting functions and secretion of pro-inflammatory cytokines (e.g. TNF $\alpha$, IL-1 $\beta$, IL-6) [36, 38]. Alternatively, the M2 phenotype expands in response to Th2-derived IL-4 and IL-13 [39], but cancer cell-derived macrophage-colony stimulating factor (M-CSF) also promotes M2 polarization by binding CSF1 receptor (CSF1-R). M2 macrophages express antiinflammatory cytokines (e.g. IL-10, CCL22, CCL18) and 
low levels of IL-12, thereby exerting anti-inflammatory, angiogenic and pro-tumoral effects [36]. Impeding M2 polarization to promote anti-tumor immune responses has gained clinical interest (e.g. CSF1 inhibition) and also preclinical studies of genetic TAM reprogramming are promising [40, 41].

\section{Cancer-associated fibroblasts}

Cancer associated fibroblasts (CAF) constitute one of the most prominent, yet highly heterogenous components of the TME. They express a variety of molecular markers, e.g. $\alpha$-SMA, S100A4, FAP, PDGFR $\alpha / \beta$, none of which, however, is unique for the fibroblast lineage. Next to immune cells CAFs have emerged as important mediators of the complex stroma-tumor interactions, promoting local immunosuppression and orchestrating immune cell trafficking [42]. CAFs may express PD-L1 (e.g. upon IFN- $\gamma$ ) (Fig. 2) but may also promote PD-L1 expression on tumor cells via cytokine secretion (e.g. CXCL5, CXCL2) [43]. Further knowledge on CAF functionality might unveil insights in IO sensitivity.

\section{Tumor endothelial cells}

Tumor endothelial cells (TEC) have immunomodulatory functions by controlling immune cell transmigration, lymphocyte activation and function. They hold a "sentinel" role in detecting foreign antigens as antigen (cross)presenting cells, though this has been studied extensively in non-malignant inflammation and less in TEC $[44,45]$. TEC are strategically positioned at the blood-TME interface, serving as "immune gatekeepers" by controlling immune cell trafficking. In NSCLC, TEC may express PD-L1 (Fig. 2) and downregulate inflammatory pathways (e.g. antigen presentation, chemotaxis, immune cell homing) [2]. On the single cell level, Goveia et al. identified distinct lung TEC subpopulations carrying the transcriptome signature of HEVs and semi-professional APCs, suggesting a role in tumor immune surveillance. Specific TEC subtypes were associated with prognosis and response to anti-angiogenic therapy [46].

\section{Resistance mechanisms}

It remains to be answered why some patients attain sustained durable IO therapy response while others evolve primary or secondary resistance. The mode of action is definitely multifactorial and includes intrinsic (e.g. cell signaling, immune recognition, gene expression, DNA damage response) and extrinsic (e.g. T cell activation, neo-angiogenesis) mechanisms [47]. The following sections briefly address relevant resistance mechanisms, many of which are already used as targets of novel therapeutic strategies as to overcome resistance (see Fig. 1).

\section{Intrinsic cancer cell resistance: immunogenicity}

Neo-antigen burden of cancer cells markedly determines tumor immunogenicity, which enhances ICI efficacy. Hence, low tumor immunogenicity may cause primary IO resistance. Immune-cancer cell interactions can promote the evolution of low-immunogenic and lowantigenic tumor subclones, a process named immunoediting [48]. Genetic instability due to impaired DNA repair can enhance tumor immunogenicity, which is the target of later discussed PARP inhibitors [47].

\section{Intrinsic $T$ cell resistance: Immuno-adaption}

In response to PD-1/PD-L1/CTLA-4 inhibition, $\mathrm{T}$ cells can upregulate alternative ICs, including $\mathrm{T}$ cell immunoglobin mucin-3 (TIM-3) or lymphocyte activation gene 3 (LAG-3), as adaptive resistance mechanism $[49,50]$. Coexpression of multiple ICs associates with severe $\mathrm{T}$ cell exhaustion, consequently leading to IO resistance [51].

\section{Extrinsic resistance: Treg and MDSCs}

An immunosuppressive TME facilitates tumor cell growth and tumor infiltrating Treg and MDSC are key players in sustaining this immunosuppression [52]. IO efficacy has been linked to lower Treg and MDSC infiltration in preclinical studies [53-55]. Moreover, Indoleamine 2,3-dioxygenase (IDO) represents an important promotor of Treg and MDSC proliferation/activation [56].

\section{Extrinsic resistance: the chemokine milieu}

Chemokines mediate immune cell recruitment into the TME and directly impact cancer and endothelial cells to regulate tumor cell proliferation, neo-angiogenesis and hence cancer progression. Multiple chemokines (four major subgroups $\mathrm{CC}, \mathrm{CXC}, \mathrm{CX} 3 \mathrm{C}, \mathrm{C}$ ) have been identified with multi-faceted roles, acting both pro- or anticancerogenic in different tumor entities. Their impact on IO resistance and efficacy remains unclear [57].

\section{Extrinsic resistance: VEGF}

Vascular endothelial growth factor (VEGF) expression within the TME is heterogenous (Fig. 2) and mainly hypoxia-driven. VEGF is the key driver of tumor neoangiogenesis but also exerts immunosuppressive effects [58]. Accordingly, anti-PD-1 non-responders showed higher VEGF levels compared to responders, suggesting a potential role of VEGF in IO resistance [59]. This at least partly explains potential additive and even synergistic effects of anti-VEGF and IO strategies, as described later.

\section{Future IO treatment strategies}

The treatment landscape of non-oncogene driven NSCLC has changed dramatically in recent years and IO is an important cornerstone of front- and later-line therapies (we refer to the latest ESMO and ASCO guidelines $[60,61])$. 
Yet, IO resistance occurs frequently, thus stressing the need for better therapy allocation based on predictive biomarkers. The cellular and molecular heterogeneity of the TME sets the stage for innovative prediction models in diagnostics and depicts a pivotal target of many tailored therapy approaches that aim to overcome IO resistance.

Multiple clinical trials in different cancer types are based on an exploding number of preclinical studies using novel IO combinations or targeted therapies. The following section will discuss the background, mode of action and clinical update of the most relevant upcoming treatment options in IO-refractory NSCLC.

\section{0 combination or re-challenge}

IC co-inhibition, by expanding the anti-PD-1 or PD-L1 backbone with a second ICI has been one of the first strategies to overcome IO resistance and most clinical experience has been gathered with combinational CTLA-4 inhibitor. The observed synergistic effect of PD-1/CTLA-4 inhibitors likely depends on the distinct patterns of PD-1 and CTLA4 in immune activation, as PD1 blockade inhibits peripheral and CTLA4-blockade central tolerance see 2.1, 3 .

\section{Clinical experience of 10 combination}

The combination of CTLA-4 and PD-1 inhibitors is effective in melanoma [62] and renal cell carcinoma (RCC) [63] patients, having led to to FDA approval. In NSCLC, CheckMate227 demonstrated a prolonged OS benefit for first-line ipilimumab plus nivolumab in advanced-stage disease (median OS 17.1 vs. 13.9 months with chemotherapy, 2-year OS of $40 \%$ vs. $32.8 \%$ (HR $0.79,97.72 \%$ CI $0.65-0.96 ; P=0.007)$ ), independent of $\mathrm{TMB}$ or PD-L1 expression. Intriguingly, the OS effect was most prominent in PD-L1 ${ }^{\text {low }}$ patients. Treatmentrelated serious adverse events (AE) of any grade were more frequent with ipilimumab plus nivolumab than with chemotherapy (24.5\% vs. $13.9 \%)$ [27].

Recent results from the phase II CITYSCAPE trial showed a significant PFS and ORR benefit for the firstline combination of the TIGIT-inhibitor see 3.1.4 tiragolumab plus atezolizumab compared to atezolizumab monotherapy in PD-L1 positive metastatic NSCLC patients. Particularly, a meaningful ORR improvement was seen in PD-L1 $1^{\text {high }}$ (TPS $>50 \%$ ) expressing patients (55.2\% vs $17.2 \%$ ) [64], while toxicity was not aggravated.

These data emphasize the potency of IO combination, but optimal patient selection criteria are still lacking.

\section{0 re-challenge}

In recent years, the dogma of disease progression being synonymous for drug resistance has been questioned [65], therefore re-challenging IO after progression displays a possible strategy.
Retrospective studies have investigated IO re-challenge in a small number of NSCLC patients with clinical benefit in only a minority of them [66-69]. Recently, a retrospective study including 10.452 NSCLC patients demonstrated the effectiveness of nivolumab retreatment after either treatment interruption or interim chemotherapy. OS in the retreatment situation significantly correlated with duration of initial IO exposure, which may be due to a time-dependent consolidation of an immune memory. The median OS for IO retreatment was above 12 months, which compares favorably with OS during initial nivolumab treatment or with standard third-line chemotherapy in advanced NSCLC [70]. Moreover, the phase III KEYNOTE-024 trial demonstrated the feasibility of a second course pembrolizumab in 10 NSCLC patients who had progressed after completion of 2 year pembrolizumab monotherapy, with an objective response rate (ORR) in 7/10 patients [71].

The question of dual ICI following IO progression has currently been investigated in two RCC studies. A small retrospective study $(n=17)$ could not show a substantial benefit of nivolumab plus ipilimumab after progression on first-line nivolumab [72]. Contrarily, the phase II TITAN trial $(n=207)$ showed a significant ORR benefit for the "immunotherapeutic boost" with 2-4 cycles of nivolumab plus ipilimumab in the first-line as compared to nivolumab monotherapy [73].

\section{0 beyond progression}

The discussion of continuing IO therapy beyond progression originates from the observation of initial pseudoprogression preceding objective response. However, pseudoprogression is rare (less than $10 \%$ of NSCLC patients) and hence IO continuation should only be considered in patients with clinical benefit and lack of severe AE [74]. Some NSCLC patients treated with ICI might present with dissociated response, where some tumor areas progress while others regress. Similarly to oligometastatic disease, a concomitant local treatment approach (radiotherapy, surgery) of the resistant clones could be discussed as possible option [75].

\section{Alternative immune checkpoints: LAG-3, TIM-3 and TIGIT}

Apart from PD-1/PD-L1/CTLA-4, other inhibitory IC regulate $\mathrm{T}$ cell response and might influence $\mathrm{IO}$ resistance mechanism. Blocking these additional IC has proven highly efficient in preclinical and clinical studies as monotherapy or in combination with PD-1/PD-L1 inhibitors. The following IC have been investigated:

Lymphocyte activation gene 3 (LAG-3 or CD223) is expressed on various immune cells (Fig. 2). LAG-3 positive $\mathrm{T}$ cells bind to ligands such as FGL1 expressed by cancer cells [76], which inhibits activation and cytokine secretion via indirectly blocking of TCR signaling [77]. 
Studies showed significant co-expression of LAG-3 and PD-1 on TILs [78, 79], with PD-1 marking a range of exhaustion phenotypes in $\mathrm{T}$ cells, from mild to anergic, while LAG-3 predominantly marks severely exhausted PD-1 positive $\mathrm{CD} 8^{+} \mathrm{T}$ cells. Hence, LAG-3 synergizes with other IC, particularly PD-1, and dual IC blockage with an anti-LAG3 antibody (e.g. IMP321, relatlimab) plus a PD-1/PD-L1 inhibitor has revealed promising preclinical results in different tumor entities and numerous clinical phase I/II trials are currently ongoing [77]. A melanoma study (NCT01968109) presented preliminary efficacy of relatlimab plus nivolumab in LAG-3 positive tumors after progression on PD-1/PD-L1 inhibitors. Further phase I/II studies in NSCLC are ongoing as upfront IO combination or in the resistance situation (NCT02750514, NCT02817633).

Similar to LAG-3, the T cell immunoglobulin mucin-3 (TIM-3) negatively regulates $\mathrm{T}$ cell activation (Fig. 2). Even though TIM-3 biology is context-dependent, TIM3 acts as an IC in severely exhausted $\mathrm{CD} 8^{+} \mathrm{T}$ cells. Here, TIM-3 ligands such as galectin-9, HMGB1 or CEACAM-1, expressed by cancer cells, activate TIM-3 and promote $\mathrm{T}$ cell anergy $[80,81]$. Based on positive preclinical results for anti-TIM-3 antibodies, several clinical trials are ongoing, testing anti-TIM-3 monotherapy or in combination with PD-1/PD-L1 inhibitors [82]: Preliminary results from the phase I Amber trial (NCT02817633) testing anti-TIM3 antibody TSR-022 in combination with a PD-1 inhibitor showed increased clinical activity in anti-PD-1 refractory NSCLC and melanoma. A phase I trial (NCT03099109) investigating anti-TIM3 antibody LY3321367 monotherapy showed preliminary anti-tumor activity and a phase I trial (NCT03708328) investigates a bi-specific antibody targeting TIM-3 and PD-1 in advanced or metastatic solid tumors.

Lastly, T cell immunoglobulin (Ig) and immunoreceptor tyrosine-based inhibitory motif (ITIM) domains (TIGIT) is a lymphocyte-specific transmembrane glycoprotein receptor (Fig. 2). As a co-inhibitory receptor, it exerts direct immunosuppressive effects on these cells through binding to CD155 (and with less affinity CD112) on APC or target cells. TIGIT is weakly expressed in naïve cells but can be rapidly induced in response to inflammatory stimuli [83]. It has been shown to impact many steps of the cancer immunity cycle (reviewed in [83]) and TIGIT inhibition can enhance anti-tumor $\mathrm{T}$ cell responses (CITYSCAPE trial), as discussed in later.

\section{0 combined with Anti-Angiogenic Drugs (AAD) Background and rationale for the combination}

VEGF is the key promoter of hypoxia-driven neoangiogenesis in the TME and also serves as important immunosuppressive molecule. Furthermore, VEGF inhibition has the ability to normalize tumor vasculature and restore chaotic blood flow, thus reducing tumor hypoxia and facilitating immune cell infiltration [84]. These mechanisms depict the functional basis of synergistic AAD and IO effects. Positive preclinical investigations in different cancer entities build a strong rationale for further clinical studies.

\section{Clinical translation}

Therapeutic combinations of AAD and IO have already been approved for RCC and endometrial cancer. In nonsquamous NSCLC, the IMpower150 trial showed an OS benefit for the first-line quadruple (atezolizumab/bevacicumab/carboplatin/paclitaxel) therapy versus $\mathrm{AAD} /$ doublet-chemotherapy with a particular benefit in patients with EGFR-mutant/ALK-positive tumors or baseline hepatic metastases [85]. The observed benefit in patients with liver metastasis adds on to previous investigations by Sandler et al. [86] that showed benefit of the AAD/chemotherapy combination, suggesting an organotypic vascular phenotype predisposing to AAD sensitivity. To clinically validate these combinational approaches, deeper investigation of synergistic anti-tumor functions and related toxicity is required. Regarding currently ongoing studies and the basic concepts we refer to other comprehensive reviews $[87,88]$.

\section{0 and radiotherapy \\ Background and rationale}

Radiation acts cytotoxic by inducing caspase-driven genomic and mitochondrial DNA fragmentation in tumor cells, promoting the release of cytochrome c from mitochondria to activate caspase 9 (CASP9) to ultimately initiate intrinsic apoptosis. Also, radiation alters the inflammatory TME by activating cytosolic DNA sensing pathways (particularly c-GAS-cGAMP-STING cascade, discussed below) in DC [89], possibly also endothelial cells (EC) [90], resulting in IFN I production and activation of anti-cancer immune responses [89]. Irradiated tumor cells often fail to activate DNA sensing pathways to produce IFN I and this barrier most likely depends on CASP9, as blocking radiation-induced CASP9 with a pan-caspase inhibitor emricasan activates tumorintrinsic type I IFN production, thereby promoting antitumor immune responses. However, in this study CASP9 inhibition resulted in PD-L1 upregulation by tumor cells as adaptive resistance strategy. Thus, combinational blockage by emricasan plus PD-L1 inhibitor enhanced radiation effects [91].

\section{Clinical translation}

The additive effect of radiotherapy and IO was investigated in the phase III PACIFIC trial. A long-term survival benefit was seen with PD-L1 inhibitor durvalumab versus placebo when used as consolidation therapy in 
patients with stage III unresectable NSCLC, who did not have disease progression after concurrent chemoradiotherapy [92].

\section{DNA damage inhibitors (PARP inhibitors) Background and rationale}

DNA damage occurs frequently during cell replication and cells have evolved various DNA Damage Response (DDR) pathways to repair damaged DNA, which when accumulating would lead to cell cycle arrest or apoptosis [93]. One DDR mechanisms involves the poly ADP-ribose polymerase (PARP), a key protein repairing single-strand DNA breakages. Therapeutic PARP inhibition triggers effective anti-cancer immune responses [94]. Double-strand DNA breaks are repaired by homologous recombination (HR). The germline BRCA1/2 genes are involved in HR mechanism and their mutation may lead to HR deficiency (HRD) [95]. HRD alone does not always induce apoptosis as other repair mechanisms can prohibit accumulation of damaged DNA. However, impairing two DDR mechanisms by adding PARPi to HR-deficient cells can lead to cell death (synthetic lethality) [95].

\section{Clinical translation}

PARP inhibitors (PARPi) are well established in the treatment of BRCA-mutated breast (Olaparib, Talazoparib) and ovarian cancer independent of HRD status (Olaparib, Niraparib, Rucaparib), being highly associated with sensitivity to platinum-based chemotherapy [96].

The BRCA-proficient NSCLC is not clinically responsive to PARPi monotherapy. However, numerous clinical studies showed synergistic effects of PARPi and IO in several solid BRCA-proficient malignancies [97]. As observed preclinically, PARPi induces genetic instability, increases TMB and neoantigen burden via DDR deficiency and may be involved in PD-L1 upregulation by cancer cells [97, 98]. This enhanced tumor immunogenicity explaining potential synergy with IO $[97,99,100]$.

Following these encouraging investigations, combinational IO/PARPi NSCLC studies are ongoing: The phase II Hudson umbrella trial (NCT03334617) investigates durvalumab plus olaparib in PD-1/PDL-1 refractory patients. The phase II Jasper trial (NCT03308942) studies first-line Niraparib plus a PD-1 inhibitor in PD-L1 positive patients progressive on chemotherapy. Results have not been released, however preliminary data from other tumor entities are promising [101, 102]. Lastly, an ongoing phase III trial (NCT02106546) investigates first-line veliparib plus chemotherapy versus placebo plus chemotherapy in advanced or metastatic NSCLC patients.

Altogether, combining PD-1/PD-L1 inhibitors with PARPi is preclinically active in BRCA-proficient tumors and numerous clinical investigations in NSCLC are ongoing.

\section{STING agonists}

\section{Background and rationale}

The cGAS-STING pathway has been identified as key intracellular pathway bridging anti-cancer innate and adaptive immunity [103]. Stimulator of Interferon Genes (STING) is a cytosolic protein of phagocytic immune, endothelial and cancer cells (Fig. 2) that gets activated by the enzyme cyclic-GMP-AMP synthase (cGAS) via the cyclic dinucleotide $(\mathrm{CDN})$ second messenger cGAMP. The STING pathway senses cytosolic DNA (self or foreign e.g. cancer-derived DNA) and, via activation of numerous downstream signals, induces IFN I IFN- $\beta$. IFN- $\beta$ plays a major role in priming adaptive immunity, including activation and recruitment of $\mathrm{CD}^{+} \mathrm{T}$ cells and promoting DC migration and maturation, thus enhancing anti-tumor immune responses [103, 104]. Cancer cells can downregulate STING activity to evade immune-mediated apoptosis [105].

\section{Clinical translation}

Based on this understanding, STING agonists, including STING-binding molecules and CDN derivatives, are being developed as novel cancer therapeutics. Preclinical studies showed dramatic anti-cancer effects of intratumorally (i.t.) applied STING agonist [90, 106-108]. Importantly, the STING induced increase in $\mathrm{CD}^{+} \mathrm{T}$ cells at the tumor site can enhance concomitant anti-PD-1 therapy effect $[109,110]$. The synthetic STING agonist $A D U$ S100 is currently under investigation in clinical phase I/II trials (NCT02675439, NCT03937141) as i.t. monotherapy or in combination with ICI in advanced solid tumors or lymphoma. A first-in-human study (NCT03010176) of STING agonist MK1454 as i.t. monotherapy or together with pembrolizumab in advanced solid tumors or lymphomas showed encouraging results with PR in $24 \%$ of patients and substantial tumor size reduction $(83 \%$ of both injected and non-injected target lesions).

In conclusion, i.t. STING agonists may evolve as potent combination to ICI treatment by "boosting" cancerdirected immune responses and sensitizing tumor cells to ICI.

\section{IDO inhibitors \\ Background and rationale}

Tryptophan catabolism, involving the key enzymes indoleamine 2,3-dioxygenase 1 and 2 (IDO1 and 2) and tryptophan-2,3-dioxygenase (TDO2) is a critical metabolic pathway in cancer progression. IDO is IFNinduced in cancer, stromal non-immune and immune cells that metabolizes tryptophan to kynurenine. Its overexpression has immunosuppressive functions by depleting tryptophan and increasing kynurenine in the TME. Indeed, kynurenine accumulation and tryptophan depletion promotes the generation of Tregs and MDSCs, 
and inhibits $\mathrm{T}_{\text {eff }}$ proliferation and activation [111]. IDO1 upregulation has been demonstrated in numerous cancer types, including NSCLC, and associates with poor prognosis and IO resistance [56]. Various preclinical studies demonstrated increased $\mathrm{T}$ cell proliferation and tumor infiltration as well as IL-2 upregulation upon IDO1 inhibition (reviewed in [112]). Although investigated to a lesser extent, TDO2 exerts similar immunosuppressive functions and enhanced expression has been shown in NSCLC [56].

\section{Clinical translation}

IDO1 inhibitors (IDO1i) have been tested in multiple phase I/II trials in combination with PD-1/PD-L1/ CTLA-4 inhibitors with promising results (reviewed in [113]). However, the first large phase III ECHO-301 trial evaluating the selective IDO1i epacadostat in combination with pembrolizumab in advanced melanoma was terminated early as the primary endpoint (improved PFS compared to pembrolizumab) was not reached [114]. Many flaws, such as insufficient dosing, lack of pharmacodynamic surrogates for drug efficacy and testing in an unselected patient population (without prior IDO testing) limit the value of the trial. Moreover, the inclusion of patients pre-treated with CTLA4- or BRAF inhibitors might explain the beneficial lack of selective IDO1i, as these therapies enhance TME levels of IDO1 and the compensatory molecules TDO2 and IDO2, which may have increased cytotoxic TIL and IFN- $\gamma$, hence impeding the effect of concomitant PD-1 blockade [56]. Still, the scientific rationale of IDO1i is solidly grounded and further clinical investigation is ongoing. Other drug combinations might evolve as efficient partners for IDO1i, e.g. CTLA-4 inhibitors, STING agonists or radiochemotherapy [115].

\section{Arginase inhibitors Background and rationale}

Arginine is a semi-essential amino acid critical for lymphocyte proliferation and function. The enzymes arginase 1 and 2 (ARG1/2) regulate extracellular arginine availability by converting arginine to ornithine and urea. High ARG1/2 expression and activity has been shown in various cancer types including NSCLC [116] and associates with poor prognosis. Within the TME, ARG is mainly produced by myeloid cells (i.e. MDSC, macrophages) in response to local stimuli (e.g. immunosuppressive cytokines, hypoxia, acidosis). ARG impedes $\mathrm{T}$ cell function e.g. by downregulation of TCR CD3 $\zeta$ chain, lowers Th1 cytokine production (IFN- $\gamma$, TNF- $\beta$ ) and inhibits $\mathrm{T}$ cell proliferation and differentiation [117]. Thus, therapeutic ARG inhibition may enhance anti-tumor immunity. Contrarily, preclinical studies implicated that arginine deprivation by using recombinant human ARG can induce apoptosis in some tumors, including NSCLC.

\section{Clinical translation}

ARG inhibitors have entered clinical trials and most substances competitively target ARG1 and ARG2. In advanced or metastatic solid cancers including NSCLC a phase I/II study (NCT02903914) investigates the small molecule INCB001158 alone or in combination with pembrolizumab. First results from CRC show manageable AEs and clinical responses. The substance OATD02 is a selective ARG1/2 inhibitor and has shown significant anti-tumor immunity in preclinical tumor models alone or in combination with PD-1 or IDO1i.

\section{Epigenetic modulators +10 Background and rationale}

Epigenetic-modulating drugs like 5-azacitidin (DNA hypomethylating agent) and entinostat (class I HDAC inhibitor) are well established in hematology. In addition to reactivating expression of epigenetically silenced tumor suppressor genes in cancer cells, these drugs may also selectively inhibit MDSC by induction of viral mimicry via inducing retrotransposon-derived dsRNA. This increases tumor foreignness through enhanced neoepitope expression, as well as it upregulates genes related to immune-evasion, such as B2M. In preclinical models, the combination of epigenetic modulators and PD-1 inhibitors has shown major therapeutic effects $[54,118]$.

\section{Clinical translation}

Based on these investigations, numerous phase I/II clinical trials in various solid tumor entities have been initiated, including NSCLC. Though interim analysis (e.g. ENCORE 601 trial) showed promising results, most of these studies are currently still ongoing [119].

\section{Adenosin-signaling pathway (CD73) Background and rationale}

Adenosine is an effective endogenous immunosuppressive mediator in normal and cancerous tissues. It gets either excreted by stressed or injured cells or generated via a multi-staged pathway from extracellular adenosinetriphosphate (ATP) through dephosphorylation of adenosine-monophosphate (AMP) by the enzyme CD73 [120]. In the TME both CD73 and adenosine are widely expressed on a variety of cells (Fig. 2). Adenosine acts via binding the A2a receptor (A2aR) (expressed on lymphocytes, myeloid and NK cells, CAF, EC), provoking i.e. Treg and MDSC accumulation, $\mathrm{T}_{\text {eff }}$ and NK cell inhibition or CAF proliferation, thereby fostering a tumorigenic TME. CD73 expression and consequently adenosine generation is regulated via complex molecular pathways, including HIF-1alpha, MAPK, mTOR, TGF- 
beta [120]. Some tumors overexpress CD73 as a possible immune-evading strategy while others do not. CD73 upregulation has been associated with an inferior outcome in NSCLC [121], and in preclinical cancer models, high CD73 expression correlated with a better response to CD73 blockade [122]. In NSCLC, high A2aR expression correlated with lower $\mathrm{CD}^{+}$and $\mathrm{CD}^{+} \mathrm{T}$ cell activation and lower PD-L1 expression [123].

\section{Clinical translation}

Therapeutic attempts have focused on inhibiting adenosine production by targeting CD73 or interfering with adenosine signaling by targeting A2aR. Different anti-CD73 antibodies have entered clinical trials as monotherapy or in combination with ICI: The anti-CD73 antibody oleclumab plus durvalumab is being tested in phase II studies in locally advanced or metastatic ICI-refractory (COAST, NCT03822351; HUDSON, NCT03334617, respectively) or as neo-adjuvant therapy in resectable (NeoCOAST, NCT03794544) NSCLC. Concerning A2aR antagonists the two oral small molecules cifroadenant (CPI-444) and AZD4635 are currently under investigation in phase I studies (NCT03337698 and NCT02740985, respectively) alone or in combination with PD-L1 inhibitors. NSCLC-regarding results of both studies have not been released yet.

\section{Chemokine receptor antagonists: CCR4 and CXCR2 inhibitors \\ Background and rationale}

The CC chemokine receptor type 4 (CCR4) is expressed on Treg and other circulating/tumor-infiltrating $\mathrm{T}$ cells and binding of TME-derived ligands (CCL17, CCL22) to CCR4 promotes recruitment of immunosuppressive Treg. Therapeutic Treg depletion may alleviate the suppression of anti-tumor immunity and hence synergize with PD-1 inhibition, as also suggested by a preclinical study [55]. Furthermore, the CXCL5/CXCR2-axis mediates myeloid cell recruitment and CXCR2 blockade significantly reduced presence of MDSC in murine tumors [124]. CCR4 and CXCL5 expression has been associated with poor prognosis in various cancer types including NSCLC $[125,126]$.

\section{Clinical translation}

The monoclonal anti-CCR4 antibody mogamulizumab exerts Treg-depleting effects and is FDA-approved for refractory $\mathrm{T}$ cell lymphoma. First results from phase I solid tumor trials in combination with PD-1/PD-L1/ CTLA-4 inhibitors suggest an acceptable safety profile $[127,128]$ and antitumor effects of mogamulizumab/ nivolumab in a small NSCLC subgroup [127]. Different CXCR2 antagonists are getting investigated preclinically and clinically (reviewed in [124]), acting as neutrophil- directed immunotherapy. A phase II trial is currently testing the selective CXCR2 antagonist navarixin (MK7123) together with pembrolizumab in advanced solid tumors including NSCLC (NCT03473925). Although only at the beginning of an understanding, these data pinpoint to possible future chemokine-targeted therapies in cancer.

\section{CSF1R antagonists}

\section{Background and rationale}

Polarization of TAM to the pro-tumorigenic M2 phenotype is promoted by binding of tumor cell-derived $\mathrm{M}$ CSF to CSF1R on TAM. Anti-CSF1R antibodies can deplete TAM, however clinical studies failed to show potent anti-tumor effects of the monotherapy (e.g. NCT01494688). A study by Kumar et al. showed that CSF downregulates granulocytic chemokine (e.g. CXCL1/2) production by CAF and that anti-CSF1 antibodies hence promote TME infiltration by immunosuppressive MDSC. Inhibition of both CSF1R and CXCR2 decreased TME infiltration by TAM and MDSC, significantly reduced tumor growth and enhanced the effect of PD-1 inhibitor [129].

\section{Clinical translation}

Numerous ongoing preclinical studies are testing CSF1R antagonists with different IO partners. In advanced NSCLC, two phase I trials (NCT03502330, NCT02526017) are currently investigating the CSF1R antagonist cabiralizumab in combination with an anti-CD40 $\mathrm{mAb}$ or nivolumab, respectively. Unfortunately, a recent phase II trial (NCT03336216) testing cabiralizumab plus nivolumab in advanced pancreatic cancer failed its primary endpoint.

\section{RIG-I}

\section{Background and rationale}

Retinoic acid Inducible Gene 1 (RIG-I) is a cytosolic RNA receptor ubiquitously expressed in most human body cells and is known for its major role in antiviral immune defense by inducing pyroptosis. RIG-I is also expressed in cancer cells, acting pro-inflammatory by expressing INF I and other cytokines [130]. In preclinical models, systemically applied RIG-I agonists were able to inhibit tumor growth via induction of immunogenic cancer cell death [131-133].

\section{Clinical translation}

Intratumoral application of the selective RIG-I agonist RGT100 was investigated in a small phase I/II first-inhuman study (NCT03065023) in advanced or recurrent cancer $(n=15)$. There were no dose-limiting toxicities, especially as only minimal systemic exposure was found after i.t. application. Interestingly, systemic chemokine elevation and INF-associated gene expression were 
detected. RIG-I agonists are only at the starting point of clinical applicability. Therapeutic challenges include the development of highly selective agonists due to ubiquitous RIG-I expression and to avoid uncontrolled cytokine release.

\section{Fibroblast Activation Protein (FAPa) Background and rationale}

The immunosuppressive activity of CAF can be hampered by blocking cell surface markers and most experience has been gathered with fibroblast-activation protein $\alpha(\mathrm{FAP} \alpha)$, a common but non-selective CAF marker in many cancer types [134]. In a mouse model, FAP $\alpha$ blockade resulted in tumor growth inhibition and stromal reduction of myofibroblasts and vasculature in lung and colon tumors [135]. Other preclinical strategies include FAP $\alpha$-targeted oncolytic adenovirus-vaccination [136] or FAP $\alpha$-targeted chimeric antigen receptor $\mathrm{T}$ cell (CAR-T) [137].

\section{Clinical translation}

A recent pioneer study investigated the use of a bispecific antibody (RO6874281) consisting of an interleukin-2 variant (IL-2v) domain that binds the IL-2 receptor on immune cells and a FAP $\alpha$-specific domain, which tracks the antibody-drug conjugate inside the tumor and reduces efflux. RO6874281 showed an acceptable safety profile and displayed monotherapy activity in tumor types not previously reported to respond to IL-2 [138] A phase II trial (NCT02627274) of RO6874281 together with atezolizumab is currently ongoing. CAFs and their immunosuppressive network present an interesting therapeutic target, however non-specificity of molecular markers incorporates a major hurdle and needs further exploration.

\section{Discussion}

In this article, we discussed relevant immunomodulatory pathways imprinted within the TME that fundamentally impact the evolution of IO resistance in NSCLC and summarized novel therapy approaches targeting many of these alterations. Considering that the majority of NSCLC patients eventually progress on IO therapy, combinational or multimodal treatment approaches are an unmet medical need.

The mechanisms underlying IO efficacy are still incompletely understood. Factors such as the dynamic cellular composition and heterogeneity of immunogenic and metabolic pathways within the TME, as well as the mutational load driving tumor immunogenicity, all contribute to IO effectiveness and evolution of resistance mechanisms.

The hallmarks of carcinogenesis are significantly influenced not only by cancer cell-intrinsic mechanisms but also by the different stromal cell populations [139]. The heterogeneity and complexity of the stromal TME and associated pathway activities and resistance patterns were particularly highlighted in lung cancer by recent high-resolution profiling [2]. However, it is likely that many of the here described TME alterations are universally apparent across different tumor entities and most preclinical studies and early-phase IO trials include several, mostly solid cancer types. At the current state of knowledge, no NSCLC-specific molecular target has been identified yet. Nevertheless, differences in the relative abundances of tumor infiltrating immune and stromal cells as well as the mutational burden do exist across different tumor entities [140].

Many of the discussed novel treatment approaches either aim to inhibit intrinsic immunosuppressive (IDO, CD73/adenosine, VEGF, CCR4, CXCR2, arginase) or promote proinflammatory/immunogenic (STING, RIG-I, PARP) pathways. Combinations of these targeted approaches with different ICI are often synergistic and may evolve as promising strategies to overcome IO resistance. Moreover, dual ICI therapy with PD-1/CTLA-4 antibodies may boost intrinsic anticancer immunity and has previously been translated into clinical OS benefit (see CheckMate227). Combinations of PD-L1 and alternative IC (e.g. LAG-3, TIM-3, TIGIT) have shown promising results in phase I trials.

Concerning biomarkers, PD-L1 is still considered the most robust biomarker in NSCLC, even though in many cases its predictive power is insufficient. Thus, the need for further, more complex biomarker-signatures that help to optimize patient selection for the different IO strategies is immense. A priori identification of resistance mechanisms in order to initiate targeted therapies upfront will depict a major challenge. In-depth tumor analysis including whole-genome sequencing, single cell RNA-sequencing, multidimensional flow cytometry or epigenetics might be implemented in the future as to find individualized treatment strategies.

\section{Conclusion}

IO therapy induces a wide range of cellular and molecular alterations in the TME and resistance mechanisms are only partially understood. However, as research is rapidly growing, numerous targets have been identified that may inhibit or override IO resistance. With positive results from many clinical trials, these novel IO combinational approaches pose a promising outlook for future therapies that improve clinical outcome and patient survival.

\section{Abbreviations}

AAD: Anti-angiogenic drugs; AE: Adverse events; AMP: Adenosinemonophosphate; ARG: Arginase; ATP: Adenosine-triphosphate; B2M: $\beta 2$ microglobulin; CAF: Cancer-associated fibroblasts; CCR4: CC chemokine 
receptor type 4; CDN: Cyclic dinucleotide; CGAS: Cyclic-GMP-AMP synthase; CRC: Colorectal cancer; CSF: Colony stimulating factor; CTLA-4: Cytotoxic T lymphocyte antigen 4; DDR: DNA Damage Response; FAPa: Fibroblastactivation protein; aHEV: High endothelial venules; HR: Homologous recombination; HRD: HR deficiency; IC: Immune checkpoints; ICI: Immune checkpoint inhibitors; IDO1: Indoleamine 2,3-dioxygenase; IFN: Interferon; IHC: Immunohistochemistry; IL-2v: Interleukin-2 variant; IO: Immunotherapy; LAG-3: Lymphocyte activation gene 3; LPS: Lipopolysaccharide; MCSF: Macrophage-colony stimulating factor; MDSC: Myeloid-derived suppressor cells; MHC: Major histocompatibility complex; MMR: Mismatch repair; NSCLC: Non-small cell lung cancer; ORR: Objective response rate; OS: Overall survival; PARP: Poly ADP-ribose polymerase; PARPi: PARP inhibitors; PD-1: Programmed death receptor 1; PD-L1: PD-ligand 1; RCC: Renal cell carcinoma; RIG1: Retinoic acid Inducible Gene 1; STING: Stimulator of Interferon Genes; TAM: Tumor associated macrophage; TCR: T cell receptor; TDO2: Tryptophan-2,3-dioxygenase; TEC: Tumor endothelial cells; TIGIT: T cell immunoglobulin and immunoreceptor tyrosinebased inhibitory motif domains; TIL: Tumor infiltrating T lymphocytes; TIM3: T cell immunoglobin mucin-3; TLR: Toll-like receptor; TLS: Tertiary lymphoid structures; TMB: Tumor mutational burden; TME: Tumor microenvironment; VEGF: Vascular endothelial growth

\section{Acknowledgements}

Not applicable.

\section{Submission declaration and verification}

This manuscript has not been published previously. It is not under consideration for publication elsewhere and its publication is approved by all authors. If accepted, it will not be published elsewhere in the same form, in English or in any other language, including electronically without the written consent of the copyright- holder.

\section{Authors' contributions}

$\mathrm{LH}$ and AP developed the concept of the manuscript; $\mathrm{LH}$ and AP drafted the manuscript; AP, DW and BT critically revised the manuscript, LH, BT and LZ developed the visualization and figures. All authors read and approved the final manuscript.

\section{Funding}

This research was solely supported by the "In Memoriam Dr. Gabriel Salzner Privatstiftung" and did not receive any other specific grant from funding agencies in the public, commercial, or not-for-profit sectors. The authors received no financial support for the research, authorship, and publication of this article.

\section{Availability of data and materials}

The datasets generated and/or analysed during the current study are available in the Scope repository, http://scope.aertslab.org/\#/Bernard_ Thienpont/*/welcome.

\section{Ethics approval and consent to participate}

Not applicable.

\section{Consent for publication}

Not applicable.

\section{Competing interests}

AP has received speaker's fees and honoraria for advisory boards from Astra Zeneca, BMS, Roche, Pfizer, Takeda and MSD. The other authors have no potential conflicts of interest to declare. No medical writer or other nonauthor was involved in the preparation of the manuscript.

\section{Author details}

'Internal Medicine V, Department of Hematology and Oncology, Medical University Innsbruck, Anichstraße 35, 6020 Innsbruck, Austria. ${ }^{2}$ Laboratory for Functional Epigenetics, Department of Human Genetics, KU Leuven, Herestraat 49, 3000 Leuven, Belgium. ${ }^{3}$ Medical Clinic III, Department of Oncology, Hematology, Immunoncology and Rheumatology, University Hospital Bonn (UKB), Sigmund-Freud-Street 25, 53127 Bonn, Germany.
Received: 31 May 2020 Accepted: 4 September 2020

Published online: 11 September 2020

\section{References}

1. Garon EB, Hellmann MD, Rizvi NA, Carcereny E, Leighl NB, Ahn MJ, et al. Five-year overall survival for patients with advanced non-small-cell lung Cancer treated with Pembrolizumab: results from the phase I KEYNOTE-001 study. J Clin Oncol. 2019;37(28):2518-27.

2. Lambrechts D, Wauters E, Boeckx B, Aibar S, Nittner D, Burton O, et al. Phenotype molding of stromal cells in the lung tumor microenvironment. Nat Med. 2018:24(8):1277-89.

3. van der Leun AM, Thommen DS, Schumacher TN. CD8. Nat Rev Cancer. 2020;20(4):218-32.

4. Buchbinder El, Desai A. CTLA-4 and PD-1 pathways: similarities, differences, and implications of their inhibition. Am J Clin Oncol. 2016;39(1):98-106.

5. Galon J, Bruni D. Approaches to treat immune hot, altered and cold tumours with combination immunotherapies. Nat Rev Drug Discov. 2019; 18(3):197-218.

6. Sautès-Fridman C, Petitprez F, Calderaro J, Fridman WH. Tertiary lymphoid structures in the era of cancer immunotherapy. Nat Rev Cancer. 2019;19(6): 307-25.

7. Helmink BA, Reddy SM, Gao J, Zhang S, Basar R, Thakur R, et al. B cells and tertiary lymphoid structures promote immunotherapy response. Nature. 2020;577(7791):549-55.

8. Martinet L, Garrido I, Filleron T, Le Guellec S, Bellard E, Fournie JJ, et al. Human solid tumors contain high endothelial venules: association with Tand B-lymphocyte infiltration and favorable prognosis in breast cancer. Cancer Res. 2011;71(17):5678-87.

9. Fridman WH, Zitvogel L, Sautès-Fridman C, Kroemer G. The immune contexture in cancer prognosis and treatment. Nat Rev Clin Oncol. 2017; 14(12):717-34.

10. Johansson-Percival A, He B, Li ZJ, Kjellén A, Russell K, Li J, et al. De novo induction of intratumoral lymphoid structures and vessel normalization enhances immunotherapy in resistant tumors. Nat Immunol. 2017;18(11): 1207-17.

11. Maldonado L, Teague JE, Morrow MP, Jotova I, Wu TC, Wang C, et al. Intramuscular therapeutic vaccination targeting HPV16 induces T cell responses that localize in mucosal lesions. Sci Transl Med. 2014;6(221): 221 ra13.

12. Lutz ER, Wu AA, Bigelow E, Sharma R, Mo G, Soares K, et al. Immunotherapy converts nonimmunogenic pancreatic tumors into immunogenic foci of immune regulation. Cancer Immunol Res. 2014;2(7):616-31.

13. DeFalco J, Harbell M, Manning-Bog A, Baia G, Scholz A, Millare B, et al. Nonprogressing cancer patients have persistent $B$ cell responses expressing shared antibody paratopes that target public tumor antigens. Clin Immunol. 2018;187:37-45.

14. Shalapour S, Font-Burgada J, Di Caro G, Zhong Z, Sanchez-Lopez E, Dhar D, et al. Immunosuppressive plasma cells impede T-cell-dependent immunogenic chemotherapy. Nature. 2015;521(7550):94-8.

15. Petitprez F, de Reyniès A, Keung EZ, Chen TW, Sun CM, Calderaro J, et al. B cells are associated with survival and immunotherapy response in sarcoma. Nature. 2020:577(7791):556-60.

16. Cabrita R, Lauss M, Sanna A, Donia M, Skaarup Larsen M, Mitra S, et al. Tertiary lymphoid structures improve immunotherapy and survival in melanoma. Nature. 2020;577(7791):561-5.

17. Rizvi NA, Hellmann MD, Snyder A, Kvistborg P, Makarov V, Havel J, et al. Cancer immunology. Mutational landscape determines sensitivity to PD-1 blockade in non-small cell lung cancer. Science. 2015;348(6230): $124-8$.

18. Chae $Y K$, Anker JF, Oh MS, Bais $P$, Namburi $S$, Agte $S$, et al. Mutations in DNA repair genes are associated with increased neoantigen burden and a distinct immunophenotype in lung squamous cell carcinoma. Sci Rep. 2019. 9(1):3235.

19. Le DT, Durham JN, Smith KN, Wang H, Bartlett BR, Aulakh LK, et al. Mismatch repair deficiency predicts response of solid tumors to PD-1 blockade. Science. 2017;357(6349):409-13.

20. del Campo AB, Kyte JA, Carretero J, Zinchencko S, Méndez R, GonzálezAseguinolaza $\mathrm{G}$, et al. Immune escape of cancer cells with beta2microglobulin loss over the course of metastatic melanoma. Int J Cancer. 2014;134(1):102-13. 
21. Pereira C, Gimenez-Xavier P, Pros E, Pajares MJ, Moro M, Gomez A, et al. Genomic profiling of patient-derived Xenografts for lung Cancer identifies Clin Cancer Res. 2017;23(12):3203-13.

22. Lawrence MS, Stojanov P, Mermel CH, Robinson JT, Garraway LA, Golub TR, et al. Discovery and saturation analysis of cancer genes across 21 tumour types. Nature. 2014;505(7484):495-501.

23. Gettinger S, Choi J, Hastings K, Truini A, Datar I, Sowell R, et al. Impaired HLA class I antigen processing and presentation as a mechanism of acquired resistance to immune checkpoint inhibitors in lung Cancer. Cancer Discov. 2017;7(12):1420-35.

24. Schoenfeld AJ, Hellmann MD. Acquired resistance to immune checkpoint inhibitors. Cancer Cell. 2020;37(4):443-55.

25. Havel JJ, Chowell D, Chan TA. The evolving landscape of biomarkers for checkpoint inhibitor immunotherapy. Nat Rev Cancer. 2019;19(3):133-50.

26. Hellmann MD, Nathanson T, Rizvi H, Creelan BC, Sanchez-Vega F, Ahuja A et al. Genomic Features of Response to Combination Immunotherapy in Patients with Advanced Non-Small-Cell Lung Cancer. Cancer Cell. 2018;33(5): 843-52 e4.

27. Hellmann MD, Paz-Ares L, Bernabe Caro R, Zurawski B, Kim SW, Carcereny

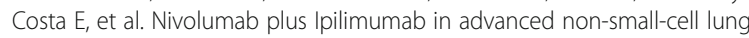
Cancer. N Engl J Med. 2019;381(21):2020-31.

28. Shukla SA, Bachireddy P, Schilling B, Galonska C, Zhan Q, Bango C, et al. Cancer-Germline Antigen Expression Discriminates Clinical Outcome to CTLA-4 Blockade. Cell. 2018;173(3):624-33 e8.

29. Riaz N, Havel JJ, Kendall SM, Makarov V, Walsh LA, Desrichard A, et al. Recurrent SERPINB3 and SERPINB4 mutations in patients who respond to anti-CTLA4 immunotherapy. Nat Genet. 2016;48(11):1327-9.

30. Zerdes I, Matikas A, Bergh J, Rassidakis GZ, Foukakis T. Genetic, transcriptional and post-translational regulation of the programmed death protein ligand 1 in cancer: biology and clinical correlations. Oncogene. 2018:37(34):4639-61.

31. Tang H, Liang Y, Anders RA, Taube JM, Qiu X, Mulgaonkar A, et al. PD-L1 on host cells is essential for PD-L1 blockade-mediated tumor regression. J Clin Invest. 2018;128(2):580-8.

32. Strauss L, Mahmoud MAA, Weaver JD, Tijaro-Ovalle NM, Christofides A, Wang Q, Pal R, Yuan M, Asara JM, Patsoukis N, et al. Targeted deletion of PD-1 in myeloid cells induces antitumor immunity. Sci Immunol. 2020;5: eaay 1863.

33. Sunshine J, Taube JM. PD-1/PD-L1 inhibitors. Curr Opin Pharmacol. 2015;23: 32-8.

34. Spigel D, de Marinis F, Giaccone G, Reinmuth $\mathrm{N}$, Vergnenegre A, Barrios $\mathrm{CH}$, et al. LBA78IMpower110: Interim overall survival (OS) analysis of a phase III study of atezolizumab (atezo) vs platinum-based chemotherapy (chemo) as first-line (1L) treatment (tx) in PD-L1-selected NSCLC. Ann Oncol. 2019; 30(Supplement_5).

35. Bensch F, van der Veen EL, Lub-de Hooge MN, Jorritsma-Smit A, Boellaard $\mathrm{R}$, Kok IC, et al. Zr-atezolizumab imaging as a non-invasive approach to assess clinical response to PD-L1 blockade in cancer. Nat Med. 2018; 24(12):1852-8.

36. Delprat V, Tellier C, Demazy C, Raes M, Feron O, Michiels C. Cycling hypoxia promotes a pro-inflammatory phenotype in macrophages via JNK/p65 signaling pathway. Sci Rep. 2020;10(1):882.

37. Palmieri EM, Gonzalez-Cotto M, Baseler WA, Davies LC, Ghesquière B, Maio $\mathrm{N}$, et al. Nitric oxide orchestrates metabolic rewiring in M1 macrophages by targeting aconitase 2 and pyruvate dehydrogenase. Nat Commun. 2020; 11(1):698

38. Yu T, Gan S, Zhu Q, Dai D, Li N, Wang H, et al. Modulation of M2 macrophage polarization by the crosstalk between Stat6 and Trim24. Nat Commun. 2019;10(1):4353.

39. Werner L, Dreyer JH, Hartmann D, Barros MHM, Büttner-Herold M, Grittner $U$, et al. Tumor-associated macrophages in classical Hodgkin lymphoma: hormetic relationship to outcome. Sci Rep. 2020;10(1):9410.

40. Zhang D, Shi R, Xiang W, Kang X, Tang B, Li C, et al. The Agpat4/LPA axis in colorectal cancer cells regulates antitumor responses via p38/p65 signaling in macrophages. Signal Transduct Target Ther. 2020;5(1):24.

41. Zhang F, Parayath NN, Ene Cl, Stephan SB, Koehne AL, Coon ME, et al. Genetic programming of macrophages to perform anti-tumor functions using targeted mRNA nanocarriers. Nat Commun. 2019;10(1):3974.

42. Liu T, Han C, Wang S, Fang P, Ma Z, Xu L, et al. Cancer-associated fibroblasts: an emerging target of anti-cancer immunotherapy. J Hematol Oncol. 2019;12(1):86
43. Teramoto K, Igarashi T, Kataoka Y, Ishida M, Hanaoka J, Sumimoto H, et al. Clinical significance of PD-L1-positive cancer-associated fibroblasts in pNOMO non-small cell lung cancer. Lung Cancer. 2019;137:56-63.

44. Hendry SA, Farnsworth RH, Solomon B, Achen MG, Stacker SA, Fox SB. The role of the tumor vasculature in the host immune response: implications for therapeutic strategies targeting the tumor microenvironment. Front Immunol. 2016;7:621.

45. Spiotto MT, Schreiber H. Rapid destruction of the tumor microenvironment by CTLS recognizing cancer-specific antigens cross-presented by stromal cells. Cancer Immun. 2005;5:8.

46. Goveia J, Rohlenova K, Taverna F, Treps L, Conradi LC, Pircher A, et al. An Integrated Gene Expression Landscape Profiling Approach to Identify Lung Tumor Endothelial Cell Heterogeneity and Angiogenic Candidates. Cancer Cell. 2020;37(1):21-36 e13.

47. Fares CM, Allen EMV, Drake CG, Allison JP, Hu-Lieskovan S. Mechanisms of resistance to immune checkpoint blockade: why does checkpoint inhibitor immunotherapy not work for all patients? Am Soc Clin Oncol Educ Book. 2019;39:147-64

48. Schreiber RD, Old LJ, Smyth MJ. Cancer immunoediting: integrating immunity's roles in cancer suppression and promotion. Science. 2011; 331(6024):1565-70.

49. Koyama S, Akbay EA, Li YY, Herter-Sprie GS, Buczkowski KA, Richards WG, et al. Adaptive resistance to therapeutic PD-1 blockade is associated with upregulation of alternative immune checkpoints. Nat Commun. 2016;7: 10501.

50. Huang RY, Francois A, McGray AR, Miliotto A, Odunsi K. Compensatory upregulation of PD-1, LAG-3, and CTLA-4 limits the efficacy of single-agent checkpoint blockade in metastatic ovarian cancer. Oncoimmunology. 2017; 6(1):e1249561.

51. Thommen DS, Schreiner J, Müller P, Herzig P, Roller A, Belousov A, et al. Progression of lung Cancer is associated with increased dysfunction of $T$ cells defined by Coexpression of multiple inhibitory receptors. Cancer Immunol Res. 2015;3(12):1344-55.

52. Gabrilovich DI, Ostrand-Rosenberg S, Bronte V. Coordinated regulation of myeloid cells by tumours. Nat Rev Immunol. 2012;12(4):253-68.

53. Highfill SL, Cui Y, Giles AJ, Smith JP, Zhang H, Morse E, et al. Disruption of CXCR2-mediated MDSC tumor trafficking enhances anti-PD1 efficacy. Sci Transl Med. 2014;6(237):237ra67.

54. Kim K, Skora AD, Li Z, Liu Q, Tam AJ, Blosser RL, et al. Eradication of metastatic mouse cancers resistant to immune checkpoint blockade by suppression of myeloid-derived cells. Proc Natl Acad Sci U S A. 2014;111(32): 11774-9.

55. Arce Vargas F, Furness AJS, Solomon I, Joshi K, Mekkaoui L, Lesko MH, et al. Fc-optimized anti-CD25 depletes tumor-infiltrating regulatory $T$ cells and synergizes with PD-1 blockade to eradicate established tumors. Immunity. 2017;46(4):577-86

56. Opitz CA, Somarribas Patterson LF, Mohapatra SR, Dewi DL, Sadik A, Platten $\mathrm{M}$, et al. The therapeutic potential of targeting tryptophan catabolism in cancer. Br J Cancer. 2020;122(1):30-44.

57. Do HTT, Lee $\mathrm{CH}$, Cho J. Chemokines and their Receptors: Multifaceted Roles in Cancer Progression and Potential Value as Cancer Prognostic Markers. Cancers (Basel). 2020;12(2).

58. Yi M, Jiao D, Qin S, Chu Q, Wu K, Li A. Synergistic effect of immune checkpoint blockade and anti-angiogenesis in cancer treatment. Mol Cancer. 2019:18(1):60.

59. Chen PL, Roh W, Reuben A, Cooper ZA, Spencer CN, Prieto PA, et al. Analysis of immune signatures in longitudinal tumor samples yields insight into biomarkers of response and mechanisms of resistance to immune checkpoint blockade. Cancer Discov. 2016;6(8):827-37.

60. Planchard D, Popat S, Kerr K, Novello S, Smit EF, Faivre-Finn C, et al. Correction to: "metastatic non-small cell lung cancer: ESMO clinical practice guidelines for diagnosis, treatment and follow-up". Ann Oncol. 2019;30(5): 863-70

61. Hanna NH, Schneider BJ, Temin S, et al. Therapy for stage IV non-small-cell lung cancer without driver alterations: ASCO and $\mathrm{OH}$ (CCO) joint guideline update. J Clin Oncol. 2020;38(14):1608-32.

62. Larkin J, Chiarion-Sileni V, Gonzalez R, Grob JJ, Rutkowski P, Lao CD, et al. Five-year survival with combined Nivolumab and Ipilimumab in advanced melanoma. N Engl J Med. 2019;381(16):1535-46.

63. Motzer RJ, Rini BI, McDermott DF, Arén Frontera O, Hammers HJ, Carducci $M A$, et al. Nivolumab plus ipilimumab versus sunitinib in first-line treatment 
for advanced renal cell carcinoma: extended follow-up of efficacy and safety results from a randomised, controlled, phase 3 trial. Lancet Oncol. 2019;20(10):1370-85.

64. Rodriguez-Abreu D, Johnson ML, Hussein MA, Cobo M, Patel AJ, Secen NM, et al. Primary analysis of a randomized, double-blind, phase II study of the anti-TIGIT antibody tiragolumab (tira) plus atezolizumab (atezo) versus placebo plus atezo as first-line (1L) treatment in patients with PD-L1selected NSCLC (CITYSCAPE). J Clin Oncol. 2020;38(15_suppl):9503.

65. Kuczynski EA, Sargent DJ, Grothey A, Kerbel RS. Drug rechallenge and treatment beyond progression--implications for drug resistance. Nat Rev Clin Oncol. 2013;10(10):571-87.

66. Fujita K, Uchida N, Kanai O, Okamura M, Nakatani K, Mio T. Retreatment with pembrolizumab in advanced non-small cell lung cancer patients previously treated with nivolumab: emerging reports of 12 cases. Cancer Chemother Pharmacol. 2018;81(6):1105-9.

67. Watanabe H, Kubo T, Ninomiya K, Kudo K, Minami D, Murakami E, et al. The effect and safety of immune checkpoint inhibitor rechallenge in non-small cell lung cancer. Jpn J Clin Oncol. 2019;49(8):762-5.

68. Katayama Y, Shimamoto T, Yamada T, Takeda T, Shiotsu S, Chihara Y, et al. Retrospective Efficacy Analysis of Immune Checkpoint Inhibitor Rechallenge in Patients with Non-Small Cell Lung Cancer. J Clin Med. 2019;9(1).

69. Niki M, Nakaya A, Kurata T, Yoshioka H, Kaneda T, Kibata K, et al. Immune checkpoint inhibitor re-challenge in patients with advanced non-small cell lung cancer. Oncotarget. 2018;9(64):32298-304.

70. Giaj Levra M, Cotté FE, Corre R, Calvet C, Gaudin AF, Penrod JR, et al. Immunotherapy rechallenge after nivolumab treatment in advanced nonsmall cell lung cancer in the real-world setting: a national data base analysis. Lung Cancer. 2020;140:99-106.

71. Reck M, Rodríguez-Abreu D, Robinson AG, Hui R, Csőszi T, Fülöp A, et al. OA14.01 KEYNOTE-024 3-Year Survival Update: Pembrolizumab vs PlatinumBased Chemotherapy for Advanced Non-Small-Cell Lung Cancer. J Thorac Oncol. 2019;14(10):S243.

72. Elias RM, Singla N, Bowman IA, Kapur P, Hannan R, Hammers H, et al. Abstract 4106: Outcomes of combined ipilimumab and nivolumab therapy following progression on nivolumab monotherapy in renal cell carcinoma: A retrospective cohort study. Cancer Res. 2019;79(13 Supplement):4106.

73. Grimm M-O, Schmidinger M, Duran Martinez I, Schinzari G, Esteban E, Schmitz M, et al. LBA57Tailored immunotherapy approach with nivolumab in advanced renal cell carcinoma (TITAN-RCC). Ann Oncol. 2019; 30(Supplement_5).

74. Borcoman E, Kanjanapan Y, Champiat S, Kato S, Servois V, Kurzrock R, et al. Novel patterns of response under immunotherapy. Ann Oncol. 2019;30(3): 385-96.

75. Tazdait M, Mezquita L, Lahmar J, Ferrara R, Bidault F, Ammari S, et al. Patterns of responses in metastatic NSCLC during PD-1 or PDL-1 inhibitor therapy: comparison of RECIST 1.1, irRECIST and RRECIST criteria. Eur J Cancer. 2018:88:38-47.

76. Wang J, Sanmamed MF, Datar I, Su TT, Ji L, Sun J, et al. Fibrinogen-like Protein 1 Is a Major Immune Inhibitory Ligand of LAG-3. Cell. 2019;176(1-2): 334-47 e12.

77. Long L, Zhang X, Chen F, Pan Q, Phiphatwatchara P, Zeng Y, et al. The promising immune checkpoint LAG-3: from tumor microenvironment to cancer immunotherapy. Genes Cancer. 2018;9(5-6):176-89.

78. He Y, Yu H, Rozeboom L, Rivard CJ, Ellison K, Dziadziuszko R, et al. LAG-3 protein expression in non-small cell lung Cancer and its relationship with PD-1/PD-L1 and tumor-infiltrating lymphocytes. J Thorac Oncol. 2017;12(5): 814-23.

79. Mishra AK, Kadoishi T, Wang X, Driver E, Chen Z, Wang XJ, et al. Squamous cell carcinomas escape immune surveillance via inducing chronic activation and exhaustion of CD8+ T cells co-expressing PD-1 and LAG-3 inhibitory receptors. Oncotarget. 2016;7(49):81341-56.

80. Barrueto L, Caminero F, Cash L, Makris C, Lamichhane P, Deshmukh RR. Resistance to checkpoint inhibition in Cancer immunotherapy. Transl Oncol. 2020;13(3):100738.

81. Das M, Zhu C, Kuchroo VK. Tim-3 and its role in regulating anti-tumor immunity. Immunol Rev. 2017;276(1):97-111.

82. Wolf $Y$, Anderson AC, Kuchroo VK. TIM3 comes of age as an inhibitory receptor. Nat Rev Immunol. 2019.

83. Manieri NA, Chiang EY, Grogan JL. TIGIT: a key inhibitor of the Cancer immunity cycle. Trends Immunol. 2017;38(1):20-8.
84. Huang Y, Yuan J, Righi E, Kamoun WS, Ancukiewicz M, Nezivar J, et al. Vascular normalizing doses of antiangiogenic treatment reprogram the immunosuppressive tumor microenvironment and enhance immunotherapy. Proc Natl Acad Sci U S A. 2012;109(43):17561-6.

85. Socinski M, Mok TSK, Nishio M, Jotte RM, Cappuzzo F, Orlandi F, et al. IMpower150 final analysis: Efficacy of atezolizumab (atezo) + bevacizumab (bev) and chemotherapy in first-line (1L) metastatic nonsquamous (nsq) non-small cell lung cancer (NSCLC) across key subgroups [abstract]. In: Proceedings of the 111th Annual Meeting of the American Association for Cancer Research; 2020 June 22-24. Philadelphia: AACR; 2020. Abstract nr CT216.

86. Sandler A, Gray R, Perry MC, Brahmer J, Schiller JH, Dowlati A, et al. Paclitaxel-carboplatin alone or with bevacizumab for non-small-cell lung cancer. N Engl J Med. 2006;355(24):2542-50.

87. Cantelmo AR, Dejos C, Kocher F, Hilbe W, Wolf D, Pircher A. Angiogenesis inhibition in non-small cell lung cancer: a critical appraisal, basic concepts and updates from American Society for Clinical Oncology 2019. Curr Opin Oncol. 2020;32(1):44-53.

88. Manegold C, Dingemans AC, Gray JE, Nakagawa K, Nicolson M, Peters S, et al. The potential of combined immunotherapy and Antiangiogenesis for the synergistic treatment of advanced NSCLC. J Thorac Oncol. 2017;12(2): 194-207.

89. Deng L, Liang H, Xu M, Yang X, Burnette B, Arina A, et al. STING-dependent cytosolic DNA sensing promotes radiation-induced type I interferondependent antitumor immunity in immunogenic tumors. Immunity. 2014; 41(5):843-52.

90. Demaria O, De Gassart A, Coso S, Gestermann N, Di Domizio J, Flatz L, et al. STING activation of tumor endothelial cells initiates spontaneous and therapeutic antitumor immunity. Proc Natl Acad Sci U S A. 2015;112(50): 15408-13.

91. Han C, Liu Z, Zhang Y, Shen A, Dong C, Zhang A, et al. Tumor cells suppress radiation-induced immunity by hijacking caspase 9 signaling. Nat Immunol. 2020;21(5):546-54

92. Gray JE, Villegas A, Daniel D, Vicente D, Murakami S, Hui R, et al. Three-year overall survival with Durvalumab after Chemoradiotherapy in stage III NSCL C-update from PACIFIC. J Thorac Oncol. 2020;15(2):288-93.

93. Jackson SP, Bartek J. The DNA-damage response in human biology and disease. Nature. 2009;461(7267):1071-8.

94. Stewart RA, Pilié PG, Yap TA. Development of PARP and immunecheckpoint inhibitor combinations. Cancer Res. 2018;78(24):6717-25.

95. da Cunha Colombo Bonadio RR, Fogace RN, Miranda VC, MDPE D. Homologous recombination deficiency in ovarian cancer: a review of its epidemiology and management. Clinics (Sao Paulo). 2018;73(suppl 1):e450s.

96. Gourley C, Balmaña J, Ledermann JA, Serra V, Dent R, Loibl S, et al. Moving from poly (ADP-ribose) polymerase inhibition to targeting DNA repair and DNA damage response in Cancer therapy. J Clin Oncol. 2019;37(25):2257-69.

97. Li A, Yi M, Qin S, Chu Q, Luo S, Wu K. Prospects for combining immune checkpoint blockade with PARP inhibition. J Hematol Oncol. 2019;12(1):98.

98. Jiao S, Xia W, Yamaguchi H, Wei Y, Chen MK, Hsu JM, et al. PARP inhibitor Upregulates PD-L1 expression and enhances Cancer-associated immunosuppression. Clin Cancer Res. 2017;23(14):3711-20.

99. Chabanon RM, Muirhead G, Krastev DB, Adam J, Morel D, Garrido M, et al. PARP inhibition enhances tumor cell-intrinsic immunity in ERCC1-deficient non-small cell lung cancer. J Clin Invest. 2019;129(3):1211-28.

100. Wang Z, Sun K, Xiao Y, Feng B, Mikule K, Ma X, et al. Niraparib activates interferon signaling and potentiates anti-PD-1 antibody efficacy in tumor models. Sci Rep. 2019;9(1):1853.

101. Karzai F, Madan RA, Owens H, Couvillon A, Hankin A, Williams M, et al. A phase 2 study of olaparib and durvalumab in metastatic castrate-resistant prostate cancer (mCRPC) in an unselected population. J Clin Oncol. 2018; 36(6_suppl):163.

102. Konstantinopoulos PA, Waggoner SE, Vidal GA, Mita MM, Fleming GF, Holloway RW, et al. TOPACIO/Keynote-162 (NCT02657889): A phase 1/2 study of niraparib + pembrolizumab in patients (pts) with advanced triplenegative breast cancer or recurrent ovarian cancer (ROC) - Results from ROC cohort. J Clin Oncol. 2018;36(15_suppl):106.

103. Li A, Yi M, Qin S, Song Y, Chu Q, Wu K. Activating CGAS-STING pathway for the optimal effect of cancer immunotherapy. J Hematol Oncol. 2019;12(1):35.

104. Zitvogel L, Galluzzi L, Kepp O, Smyth MJ, Kroemer G. Type I interferons in anticancer immunity. Nat Rev Immunol. 2015;15(7):405-14. 
105. Miao L, Qi J, Zhao Q, Wu QN, Wei DL, Wei XL, et al. Targeting the STING pathway in tumor-associated macrophages regulates innate immune sensing of gastric cancer cells. Theranostics. 2020;10(2):498-515.

106. Ohkuri T, Ghosh A, Kosaka A, Zhu J, Ikeura M, David M, et al. STING contributes to antiglioma immunity via triggering type I IFN signals in the tumor microenvironment. Cancer Immunol Res. 2014;2(12):1199-208.

107. Fu J, Kanne DB, Leong M, Glickman LH, McWhirter SM, Lemmens E, et al. STING agonist formulated cancer vaccines can cure established tumors resistant to PD-1 blockade. Sci Transl Med. 2015;7(283):283ra52.

108. Corrales L, Glickman LH, McWhirter SM, Kanne DB, Sivick KE, Katibah GE, et al. Direct activation of STING in the tumor microenvironment leads to potent and systemic tumor regression and immunity. Cell Rep. 2015;11(7):1018-30.

109. Kinkead HL, Hopkins A, Lutz E, Wu AA, Yarchoan M, Cruz K, et al. Combining STING-based neoantigen-targeted vaccine with checkpoint modulators enhances antitumor immunity in murine pancreatic cancer. JCI Insight. 2018;3(20).

110. Dorta-Estremera S, Hegde VL, Slay RB, Sun R, Yanamandra AV, Nicholas C, et al. Targeting interferon signaling and CTLA-4 enhance the therapeutic efficacy of anti-PD-1 immunotherapy in preclinical model of HPV. J Immunother Cancer. 2019;7(1):252.

111. Labadie BW, Bao R, Luke JJ. Reimagining IDO pathway inhibition in Cance immunotherapy via downstream focus on the tryptophan-Kynurenine-aryl hydrocarbon Axis. Clin Cancer Res. 2019;25(5):1462-71.

112. Yentz S, Smith D. Indoleamine 2,3-Dioxygenase (IDO) inhibition as a strategy to augment Cancer immunotherapy. BioDrugs. 2018;32(4):311-7.

113. Prendergast GC, Malachowski WP, DuHadaway JB, Muller AJ. Discovery of IDO1 inhibitors: from bench to bedside. Cancer Res. 2017;77(24):6795-811.

114. Long GV, Dummer R, Hamid O, Gajewski TF, Caglevic C, Dalle S, et al. Epacadostat plus pembrolizumab versus placebo plus pembrolizumab in patients with unresectable or metastatic melanoma (ECHO-301/KEYNOTE252): a phase 3, randomised, double-blind study. Lancet Oncol. 2019;20(8): 1083-97.

115. Muller AJ, Manfredi MG, Zakharia Y, Prendergast GC. Inhibiting IDO pathways to treat cancer: lessons from the ECHO-301 trial and beyond. Semin Immunopathol. 2019;41(1):41-8.

116. Süer Gökmen S, Yörük Y, Cakir E, Yorulmaz F, Gülen S. Arginase and ornithine, as markers in human non-small cell lung carcinoma. Cancer Biochem Biophys. 1999;17(1-2):125-31.

117. Grzywa TM, Sosnowska A, Matryba P, Rydzynska Z, Jasinski M, Nowis D, et al. Myeloid cell-derived Arginase in Cancer immune response. Front Immunol. 2020;11:938

118. Wrangle J, Wang W, Koch A, Easwaran H, Mohammad HP, Vendetti F, et al. Alterations of immune response of non-small cell lung Cancer with Azacytidine. Oncotarget. 2013;4(11):2067-79

119. Cho JH, Oezkan F, Koenig M, Otterson GA, Herman JG, He K. Epigenetic therapeutics and their impact in immunotherapy of lung Cancer. Curr Pharmacol Rep. 2017;3(6):360-73.

120. Allard D, Chrobak P, Allard B, Messaoudi N, Stagg J. Targeting the CD73adenosine axis in immuno-oncology. Immunol Lett. 2019;205:31-9.

121. Inoue $Y$, Yoshimura K, Kurabe N, Kahyo T, Kawase A, Tanahashi M, et al. Prognostic impact of CD73 and A2A adenosine receptor expression in nonsmall-cell lung cancer. Oncotarget. 2017;8(5):8738-51.

122. Turcotte M, Allard D, Mittal D, Bareche $Y$, Buisseret $L$, José $V$, et al. CD73 promotes resistance to HER2/ErbB2 antibody therapy. Cancer Res. 2017; 77(20):5652-63.

123. Viveiros P, Sukhadia B, Nunna P, Park KW, Chuang J, Zou L, et al. Abstract 523: Implications of the $\mathrm{A} 2 \mathrm{a}$ receptor (A2aR) on tumor microenvironment in nonsmall cell lung cancer (NSCLC). Cancer Res. 2019;79(13 Supplement):523.

124. Cheng Y, Ma XL, Wei YQ, Wei XW. Potential roles and targeted therapy of the CXCLs/CXCR2 axis in cancer and inflammatory diseases. Biochim Biophys Acta Rev Cancer. 2019;1871(2):289-312.

125. Liu W, Wei X, Li L, Wu X, Yan J, Yang H, et al. CCR4 mediated chemotaxis of regulatory $T$ cells suppress the activation of T cells and NK cells via TGF- $\beta$ pathway in human non-small cell lung cancer. Biochem Biophys Res Commun. 2017:488(1):196-203.

126. Wu K, Yu S, Liu Q, Bai X, Zheng X. The clinical significance of CXCL5 in nonsmall cell lung cancer. Onco Targets Ther. 2017;10:5561-73.

127. Doi T, Muro K, Ishii H, Kato T, Tsushima T, Takenoyama M, et al. A phase I study of the anti-CC chemokine receptor 4 antibody, Mogamulizumab, in combination with Nivolumab in patients with advanced or metastatic solid tumors. Clin Cancer Res. 2019;25(22):6614-22.
128. Zamarin D, Hamid O, Nayak-Kapoor A, Sahebjam S, Sznol M, Collaku A, et al. Mogamulizumab in combination with Durvalumab or Tremelimumab in patients with advanced solid tumors: a phase I study. Clin Cancer Res. 2020.

129. Kumar V, Donthireddy L, Marvel D, Condamine T, Wang F, Lavilla-Alonso S, et al. Cancer-Associated Fibroblasts Neutralize the Anti-tumor Effect of CSF1 Receptor Blockade by Inducing PMN-MDSC Infiltration of Tumors. Cancer Cell. 2017;32(5):654-68 e5.

130. Wolf D, Fiegl H, Zeimet AG, Wieser V, Marth C, Sprung S, et al. High RIG-I expression in ovarian cancer associates with an immune-escape signature and poor clinical outcome. Int J Cancer. 2020;146(7):2007-18.

131. Ellermeier J, Wei J, Duewell P, Hoves S, Stieg MR, Adunka T, et al. Therapeutic efficacy of bifunctional siRNA combining TGF- $\beta 1$ silencing with RIG-I activation in pancreatic cancer. Cancer Res. 2013;73(6):1709-20.

132. Duewell P, Steger A, Lohr H, Bourhis H, Hoelz H, Kirchleitner SV, et al. RIG-Ilike helicases induce immunogenic cell death of pancreatic cancer cells and sensitize tumors toward killing by CD8(+) T cells. Cell Death Differ. 2014; 21(12):1825-37.

133. Poeck H, Besch R, Maihoefer C, Renn M, Tormo D, Morskaya SS, et al. 5'triphosphate-siRNA: turning gene silencing and rig-I activation against melanoma. Nat Med. 2008;14(11):1256-63.

134. Ziani L, Chouaib S, Thiery J. Alteration of the antitumor immune response by Cancer-associated fibroblasts. Front Immunol. 2018:9:414.

135. Öhlund D, Elyada E, Tuveson D. Fibroblast heterogeneity in the cancer wound. J Exp Med. 2014;211(8):1503-23.

136. Zhang Y, Ertl HC. Depletion of FAP+ cells reduces immunosuppressive cells and improves metabolism and functions CD8+T cells within tumors. Oncotarget. 2016;7(17):23282-99.

137. Wang LC, Lo A, Scholler J, Sun J, Majumdar RS, Kapoor V, et al. Targeting fibroblast activation protein in tumor stroma with chimeric antigen receptor T cells can inhibit tumor growth and augment host immunity without severe toxicity. Cancer Immunol Res. 2014;2(2):154-66.

138. Soerensen MM, Ros W, Rodriguez-Ruiz ME, Robbrecht D, Rohrberg KS, Martin-Liberal J, et al. Safety, PK/PD, and anti-tumor activity of RO6874281, an engineered variant of interleukin-2 (IL-2v) targeted to tumor-associated fibroblasts via binding to fibroblast activation protein (FAP). J Clin Oncol. 2018;36(15_suppl):e15155-e.

139. Hanahan D, Coussens LM. Accessories to the crime: functions of cells recruited to the tumor microenvironment. Cancer Cell. 2012;21(3):309-22.

140. Giraldo NA, Sanchez-Salas R, Peske JD, Vano Y, Becht E, Petitprez F, et al. The clinical role of the TME in solid cancer. Br J Cancer. 2019;120(1):45-53.

\section{Publisher's Note}

Springer Nature remains neutral with regard to jurisdictional claims in published maps and institutional affiliations.

Ready to submit your research? Choose BMC and benefit from:

- fast, convenient online submission

- thorough peer review by experienced researchers in your field

- rapid publication on acceptance

- support for research data, including large and complex data types

- gold Open Access which fosters wider collaboration and increased citations

- maximum visibility for your research: over $100 \mathrm{M}$ website views per year

At $\mathrm{BMC}$, research is always in progress.

Learn more biomedcentral.com/submission 